\title{
Obstructive sleep apnea in the formerly preterm infant: an overlooked diagnosis
}

\author{
Preeti B. Sharma ${ }^{1}$, Fuad Baroody ${ }^{1,2,3}$, David Gozal $^{1}{ }^{*}$ and Lucille A. Lester ${ }^{1}$ \\ ' Section of Pediatric Pulmonary Medicine, Department of Pediatrics, Comer Children's Hospital, University of Chicago, Chicago, IL, USA \\ ${ }^{2}$ Department of Otolaryngology-Head and Neck Surgery, University of Chicago, Chicago, IL, USA \\ ${ }^{3}$ Department of Pediatrics, University of Chicago, Chicago, IL, USA
}

Edited by:

Mehmet Y. Agargün, Yuzuncu Yil

University School of Medicine, Turkey

\section{Reviewed by:}

Takashi Ono, Tokyo Medical and

Dental University, Japan

Karl AE. Karlsson, Reykjavik University,

Iceland

\section{*Correspondence:}

David Gozal, Section of Pediatric Pulmonary Medicine, 5841 South Maryland Avenue MC 4064, Chicago, IL 60637, USA.

e-mail:dgozal@peds.bsd. uchicago.edu
Background: Obstructive sleep apnea syndrome (OSA) is a frequent disorder in children. The clinical characteristics of OSA in very young children under 2 years of age, and more particularly, in those born prematurely, and who have respiratory complications such as bronchopulmonary dysplasia (BPD), are not well defined. We therefore retrospectively reviewed our experience in a group of preterm infants with OSAS. Methods: The records of premature infants with BPD followed in the Pediatric Pulmonary Clinic at the University of Chicago who were diagnosed with OSA from 2004 to 2009 were reviewed and analyzed. Results: Twelve children, eight males, and four females with a mean gestational age of 27 weeks were found to have OSA. Mean age at diagnosis was 19 months. Inability to wean nighttime oxygen, the need to resume oxygen after intercurrent respiratory illness, and snoring were the most common presenting symptoms. The apnea-hypopnea index ranged from 1 to 120/h total sleep time (TST; mean: 29). $\mathrm{SpO}_{2}$ nadir ranged from 50 to $91 \%$. Despite adenotonsillectomy (AT), all children had persistent sleep disordered breathing. Conclusion: In preterm infants, while snoring is a frequent symptom, poor weight gain, and inability to wean nighttime oxygen may indicate the need for further investigation for OSA. In the former preterm infant structural changes in the airway may play an important role along with adenotonsillar hypertrophy. A high level of clinical awareness is required to identify OSA in the formerly preterm infant.

Keywords: obstructive sleep apnea, premature infant, lung disease, tonsillectomy

\section{INTRODUCTION}

Obstructive sleep apnea syndrome (OSA) is a disorder identified by recurrent episodes of complete or partial airway obstruction during sleep that result in intermittent hypoxia, recurrent arousals, and sleep disruption (Arens and Marcus, 2004). It is most often diagnosed in children who are pre-school or school aged, with the most common, albeit non-exclusive, contributing factor being adenotonsillar hypertrophy (ATH). Other risk factors include obesity, craniofacial characteristics, and neuromuscular abnormalities (Arens and Marcus, 2004). OSA affects $1-3 \%$ of school age children (Chervin et al., 2002; Capdevila et al., 2008; Gozal, 2008), with the most common nighttime symptoms being snoring, witnessed apnea, restless sleep, nocturnal enuresis, and frequent nightmares (Chervin et al., 2002). Daytime symptoms include nasal obstruction and mouth breathing, as well as morning headache, trouble concentrating, and daytime sleepiness (Capdevila et al., 2008; Gozal, 2008).

\footnotetext{
Abbreviations: AHI, apnea-hypopnea index; ALTE, apparent life threatening event; AT, adenotonsillectomy; ATH, adenotonsillar hypertrophy; BPD, bronchopulmonary dysplasia; BW, birth weight; $\mathrm{CP}$, cerebral palsy; $\mathrm{CPAP}$, continuous positive airway pressure; FTT, failure to thrive; GA, gestational age; IVH, intraventricular hemorrhage; NICU, neonatal intensive care unit; OSA, obstructive sleep apnea syndrome; PSG, polysomnogram; PVL, periventricular leukomalacia; RDI, respiratory disturbance index.
}

The clinical presentation and overall determinants of OSA have not been thus far well characterized in children under 2 years of age, even if the frequency of habitual snoring, the hallmark symptom of OSA, is high in this age group (Montgomery-Downs and Gozal, 2006), and appears to be modulated by the breastfeeding history of the child (Montgomery-Downs et al., 2007). Interestingly, adenotonsillectomy (AT) appears to be an effective intervention in very young children with OSA (Greenfeld et al., 2003), although more studies are clearly needed to confirm such preliminary findings (Bhattacharjee et al., 2010). Furthermore, although prematurity has been identified as an important risk factor for OSA occurring later in life (Rosen et al., 2003; Paavonen et al., 2007; Hibbs et al., 2008; Calhoun et al., 2010), the clinical spectrum of OSA in infants born prematurely is unclear. A recent study by Montgomery-Downs et al. (2010) suggested that $8.1 \%$ of infants born at a mean of 31.6 weeks gestation were reported to snore frequently, a rate that is remarkably similar to the snoring prevalence reported for older children. However, this study did not differentiate between premature infants born at various postconceptional ages, and more importantly did not examine the impact of prematurity-associated respiratory complications, such as bronchopulmonary dysplasia (BPD). BPD is diagnosed when an infant still requires additional oxygen and continues to show clinical signs and symptoms after 28 days of age (or past 36 weeks post-conceptional age). In the former preterm infant with $\mathrm{BPD}$, the 
prolonged need for oxygen, the persistence of respiratory symptoms, and failure to gain weight could be erroneously attributed to the known chronic lung disease rather than to underlying OSA, and consequently be overlooked in this highly susceptible population. In this study, we have reviewed our experience in the diagnosis and treatment of preterm infants with OSA.

\section{MATERIALS AND METHODS}

The medical records of all 387 premature infants with BPD followed in the Pediatric Pulmonary Clinic at the University of Chicago from 2004 to 2009 were reviewed to identify those who were diagnosed with OSA either clinically or by overnight polysomnography (PSG). The latter records were then retrospectively analyzed after approval by the institutional review board.

\section{POLYSOMNOGRAPHY}

Patients underwent PSG study in the Sleep Laboratory at the University of Chicago Hospitals in the presence of a parent or caregiver. No medications were used to induce sleep. The following parameters were measured: chest and abdominal wall motion by inductance plethysmography, heart rate by electrocardiography, nasal air flow with pressure cannula, and an oronasal thermistor. $\mathrm{SpO}_{2}$ was assessed by pulse oximetry with simultaneous recording of the pulse waveform. Bilateral electro-oculogram, eight channels of electroencephalogram, chin, and anterior tibial electromyograms were monitored. Tracheal sound was measured with a microphone sensor. Sleep position and patient behavior was monitored by a video camera inside the room. End tidal carbon dioxide levels were not measured. All of the studies were initially scored by a certified technician, and were subsequently reviewed by physicians experienced in PSG interpretation. Sleep architecture was assessed by standard techniques (Rechtschaffen and Kales, 1968). The proportion of time spent in each sleep stage was expressed as a percentage of total sleep time (TST). Central, obstructive, and hypopnea events were counted. Obstructive events were defined as the absence of air flow with continued chest wall and abdominal movement for a duration of at least two breaths. Hypopneas were defined as a decrease in oronasal flow of greater than or equal to $50 \%$ with a corresponding decrease in oxygen saturation of $3 \%$ or more, and/or EEG arousal (Montgomery-Downs et al., 2006).

Charts were reviewed for birth history, including birth weight and gestational age, as well as neonatal intensive care course including duration of endotracheal intubation. We excluded any child with known craniofacial syndromes or any other genetic disorder. Outpatient clinic charts were used to record and assess weight gain after discharge from the neonatal intensive care unit (NICU). Polysomnography reports were reviewed, and post intervention PSGs and clinical course were analyzed as patients continued to be followed in the Pediatric Pulmonary Clinic. This study was approved by the Institutional Review Board of the University of Chicago.

Data were expressed as means. Explorative associations were conducted using linear regression approaches. Statistical analyses were performed using SPSS software (version 16.0; SPPS Inc., Chicago, IL, USA). All $p$-values reported are two-tailed with statistical significance set at $<0.05$.

\section{RESULTS}

Clinical findings are summarized in Table 1. The charts of 12 children who were followed in the Pediatric Pulmonary Clinic and known to have a history of premature birth and documented OSA were reviewed. Mean gestational age at birth was 27 weeks, ranging from 24 to 33 weeks. Birth weights were unavailable in two children who were in foster homes since NICU discharge from other hospitals. The mean birth weight of the 10 remaining children was $1,020 \mathrm{~g}(622-1,775 \mathrm{~g})$. Six of $10 \mathrm{had}$ a birth weight of less than $1,000 \mathrm{~g}$. Two children had no history of intubation in the NICU; the remaining 10 infants were intubated for a total of 3-93 days. Two infants required intubation for respiratory failure after discharge from the NICU. Patient \#1 experienced an apparent life threatening event (ALTE) at 8 months of age, and was intubated. After extubation, he had witnessed bradycardia and profound desaturations during sleep. Patient \#9 was intubated for respiratory failure due to influenza

Table 1 | Patient demographic data.

\begin{tabular}{|c|c|c|c|c|c|c|}
\hline No. & GA (weeks) & $\mathrm{BW}(\mathrm{g})$ & Time w/ETT (days) & Medical problems & Age at presentation (months) & Major presenting signs \\
\hline 1 & 27 & 1,100 & 7 & BPD & 10 & ALTE, supplemental oxygen \\
\hline 2 & 27 & 962 & 86 & $\mathrm{BPD}, \mathrm{IVH}, \mathrm{CP}$ & 28 & Snoring, FTT \\
\hline 3 & 24 & 733 & 60 & BPD, IVH, seizure & 15 & ALTE, FTT \\
\hline 4 & 24 & 890 & 91 & BPD, PVL, seizure & 20 & Inability to extubate \\
\hline 5 & 29 & 814 & 30 & BPD, IVH & 13 & Snoring \\
\hline 6 & 25 & 680 & 90 & BPD & 22 & Snoring, supplemental oxygen, FTT \\
\hline 7 & 24 & 622 & 93 & BPD & 22 & Snoring, supplemental oxygen, FTT \\
\hline 8 & 28 & 1,220 & 12 & BPD & 33 & Snoring \\
\hline 9 & 29 & 1,775 & 3 & None & 9 & Snoring, supplemental oxygen \\
\hline 10 & 29 & 1,410 & 0 & None & 18 & Snoring \\
\hline 11 & 33 & Unknown & 0 & None & 15 & Snoring \\
\hline 12 & 26 & Unknown & 60 & BPD & 15 & FTT, supplemental oxygen \\
\hline
\end{tabular}

BPD, bronchopulmonary dysplasia; IVH, intraventricular hemorrhage; CP, cerebral palsy; PVL, periventricular leukomalacia; ALTE, acute life threatening event; FTT, failure to thrive; GA, gestational age; BW, birth weight; ETT, endotracheal tube. 
A infection. He was subsequently extubated; however he presented 6 months later with cor pulmonale and respiratory failure and could not be extubated. Complications of prematurity included BPD $(n=9)$, intraventricular hemorrhage (IVH; $n=3)$, cerebral palsy $(n=1)$, periventricular leukomalacia $(n=1)$, and seizures $(n=1)$.

The mean age at the time of diagnosis with OSA was 19 months, with a range of 9-28 months. The most common presenting sign was snoring $(n=8 / 12)$. Other presenting signs included failure to gain weight appropriately $(n=5 / 12)$, persistent supplemental oxygen requirements or the need to resume supplemental oxygen $(n=5 / 12)$, inability to remain extubated after a respiratory illness requiring intubation $(n=1)$, and an $\operatorname{ALTE}(n=1)$.

Polysomnography findings are summarized in Table 2. Ten children underwent overnight PSG as a part of their diagnostic work-up. Eight of ten children had central apnea events, five had obstructive apneas, and all were noted to have significant hypopnea events. The apnea-hypopnea index (AHI) ranged $1-120 / \mathrm{h}$ TST, with a mean AHI of 29/h TST. Patient \#3 had an AHI of $1 / \mathrm{h}$ TST, but a rapid eye movement (REM) sleep-related respiratory disturbance index (RDI) of 3/h TST and $\mathrm{SpO}_{2}$ nadir of $90 \%$. All children had $\mathrm{SpO}_{2}$ nadir below $92 \%$ (Table 1 ).

All 12 patients underwent adenotonsillectomy (AT). One child was observed to have profound obstructive events in the pediatric intensive care unit after AT and ultimately required tracheotomy. Two patients continued to have severe sleep disordered breathing after AT, one required positive pressure ventilation and one required supplemental oxygen. All patients had comprehensive evaluations by a pediatric otolaryngologist prior to AT, and none had evidence of airway structural abnormality such as subglottic narrowing. Five children underwent repeat PSG postoperatively, and all but one had an improvement in AHI, however three of the five had persistent OSA as defined by AHI $\geq 5 / \mathrm{h}$ TST.

There was no significant correlation between degree of prematurity or birth weight and severity of OSA. Additionally, there were no significant associations between duration of intubation, diagnosis of IVH or seizure activity during the neonatal period, or the estimated size of adenotonsillar tissue as visually assessed by the pediatric otolaryngologist, and the frequency of either obstructive or central apneas.

\section{DISCUSSION}

This retrospective review of 12 prematurely born young children with OSA illustrates some of the overlap between prematurityassociated complications such as BPD and OSA, and the potential for such overlap to delay the diagnosis of OSA. Furthermore, we show that although adenotonsillar size is contributory to the severity of OSA in these young children, AT is insufficient to normalize their breathing patterns during sleep.

Adenotonsillar hypertrophy is the leading cause of OSA in children (Suen et al., 1995; Darrow and Siemens, 2002), with peak ATH and related OSA occurring between 3 and 6 years of age (Bhattacharjee et al., 2010). However, OSA secondary to ATH is considered fairly uncommon in infants in general, and even less characterized in infants born prematurely (Greenfeld et al., 2003; Montgomery-Downs et al., 2010). This is surprising, considering that premature infants are predisposed to upper airway obstruction and oxygen desaturations that are mediated by the underlying decreased upper airway muscle tone, high nasal resistance, and the presence of a highly compliant chest wall (Arens and Marcus, 2004). A previous study in young children ( $<18$ months) with OSA and ATH reported a greater occurrence in infants who were born preterm (Greenfeld et al., 2003), as well as a male preponderance, which is now corroborated by our current findings. Additionally, 5 of 12 patients experienced sub-optimal weight gain, a common occurrence in the series of 29 infants reported by Greenfeld et al. (2003). Although the mechanisms for such poor somatic growth are unclear, poor peripheral tissue oxygenation, disruption of growth hormone pathways, and increased energy expenditure are all likely to contribute (Marcus et al., 1994; Capdevila et al., 2008). Anorexia and dysphagia resulting from the ATH could also reduce caloric intake (Marcus et al., 1994). Of

Table 2 | Polysomnography results and treatment outcomes.

\begin{tabular}{|c|c|c|c|c|c|c|c|c|c|}
\hline & $\begin{array}{l}\text { Age at PSG } \\
\text { (months) }\end{array}$ & $\begin{array}{l}\text { Total sleep } \\
\text { time (min) }\end{array}$ & $\begin{array}{l}\text { Central } \\
\text { apneas (n) }\end{array}$ & $\begin{array}{l}\text { Obstructive } \\
\text { apneas }(n)\end{array}$ & $\begin{array}{l}\text { Hypopnea } \\
\text { events }(n)\end{array}$ & $\begin{array}{l}\text { AHI } \\
\text { (/h TST) }\end{array}$ & $\begin{array}{l}\mathrm{SpO}_{2} \\
\text { nadir }\end{array}$ & Intervention & $\begin{array}{l}\text { Follow-up } \\
\text { PSG (AHI) }\end{array}$ \\
\hline 1 & 9 & 362 & 0 & 325 & 108 & 72 & $50 \%$ & AT then tracheostomy & None \\
\hline 2 & 28 & 412 & 24 & & 80 & 15 & $86 \%$ & AT & None \\
\hline 3 & 21 & 323 & 2 & 0 & 1 & 1 & $87 \%$ & AT & 1 \\
\hline 4 & None & & & & & & & AT & None \\
\hline 5 & 12 & 374 & 6 & 0 & 35 & 7 & $92 \%$ & AT & 2 \\
\hline 6 & 25 & 391 & 0 & 0 & 44 & 7 & $83 \%$ & AT & None \\
\hline 7 & 22 & 157 & 2 & 0 & 67 & 26 & $72 \%$ & AT & None \\
\hline 8 & None & & & & & & & AT & None \\
\hline 9 & 9 & 343 & 2 & 138 & 84 & 38 & $46 \%$ & AT, CPAP & 23 \\
\hline 10 & 30 & 374 & 1 & 3 & 60 & 20 & $88 \%$ & AT & 16 \\
\hline 11 & 16 & 351 & 1 & 503 & 188 & 120 & $74 \%$ & AT, supplemental oxygen & 6 \\
\hline 12 & 17 & 322 & 4 & 32 & 164 & 39 & $81 \%$ & AT & None \\
\hline
\end{tabular}

PSG, polysomnography; AHI, apnea-hypopnea index; RDI, respiratory disturbance index; $\mathrm{SpO}_{2}$ nadir, lowest recorded oxygen saturation; AT, adenotonsillectomy; CPAP, continuous positive airway pressure; TST, total sleep time. 
note, our current assessment of adenotonsillar size as well as that performed by previous authors was not quantitative, but rather reflected an estimate of ATH in relation to airway size. More accurate measurements of upper airway lymphadenoid tissues should be implemented in the future.

Previous studies examining risk factors for OSA in schoolaged children have clearly identified prematurity, but have not shown significant correlations between the traditional markers of severity of neonatal illness, such as birth weight, IVH, BPD, and duration of ventilation, and the prevalence of OSA (Hibbs et al., 2008). Although two-thirds of the patients in our series carried a diagnosis of BPD, this is more likely accounted for the fact that our cohort included patients followed in the Pediatric Pulmonary clinic who were referred specifically for management of the respiratory complications of prematurity. Furthermore, among the 9 of 12 infants with OSA who had BPD, the severity of BPD was not correlated with severity of the OSA. Similar findings have been previously reported, whereby the presence of BPD has not effect on the frequency of obstructive respiratory events during sleep (Fajardo et al., 1993). However, we should emphasize that this is a descriptive study in a small cohort of patients, and that causal inferences will have to await larger future studies.

It should be emphasized that while degree of prematurity does not appear to correlate with the severity of OSA, the morbidities of OSA and the response to treatment may be less favorable in the formerly preterm infant. McGowen et al. (1992) found that complications related to AT were closely correlated to a history of low birth weight and prematurity. Also, the intermittent hypoxia associated with OSA can facilitate the occurrence of pulmonary vasoconstriction and increased pulmonary artery pressures, particularly in those infants with BPD (Sie et al., 1997; Capdevila et al., 2008). Sie et al. (1997) reported a case of a 2-year-old expremature infant who presented with cardiomegaly and severe right ventricular and atrial hypertrophy. He was found to have elevated pulmonary artery pressures and was noted to have had long standing snoring and markedly enlarged tonsils. It was suspected that the pulmonary hypertension and subsequent cardiac decompensation were due to OSA. Similarly, one of the patients in our series presented with respiratory failure and cor pulmonale during a viral respiratory illness. These cases highlight the fact that any young child with a history of chronic lung disease with OSA is also at risk for development or worsening of underlying pulmonary hypertension. These already compromised children may be stable until they develop a respiratory illness that

\section{REFERENCES}

Arens, R., and Marcus, C. L. (2004). Pathophysiology of upper airway obstruction: a developmental perspective. Sleep 27, 997-1019.

Bhattacharjee, R., Kheirandish-Gozal, L., Spruyt, K., Mitchell, R. B., Promchiarak, J., Simakajornboon, N., Kaditis, A. G., Splaingard, D., Splaingard, M., Brooks, L. J., Marcus, C. L., Sin, S., Arens, R., Verhulst, S. L., and Gozal, D. (2010). Adenotonsillectomy outcomes in treatment of obstructive sleep apnea in children: a multicenter retrospective study. Am. J. Respir. Crit. Care Med. 182, 676-683.

Calhoun, S. L., Vgontzas, A. N., Mayes, S. D., Tsaoussoglou, M., Sauder, K., Mahr, F., Karippot, A., Wisner, K., and Bixler, E. O. (2010). Prenatal and perinatal complications: is it the link between race and SES and childhood

may then precipitate acute cardiac decompensation (Sie et al., 1997).

Numerous possible explanations have been advanced for the higher incidence of early OSA in premature infants (Greenfeld et al., 2003; Rosen et al., 2003; Montgomery-Downs and Gozal, 2006; Paavonen et al., 2007; Hibbs et al., 2008; Calhoun et al., 2010). The elongated head shape (dolicocephaly) of children born prematurely could result in alteration of the shape and growth of the airway and contribute to upper airway narrowing thus exacerbating the probability of upper airway narrowing or collapse during sleep (McGowen et al., 1992). Also, prematurity has been linked with facial asymmetry which can also alter airway dimensions (McGowen et al., 1992). Normally in children, airway size grows proportionally to the soft tissues; however, it is not known how prematurity affects airway growth and shape, or how the prolonged presence of an endotracheal tube in the airway can alter the shape of the palate and airway growth (McGowen et al., 1992). Previous studies have postulated that preterm infants have a higher incidence of muscle hypotonia which could also predispose them to OSA (Greenfeld et al., 2003). It is also possible that there may be chronic inflammatory processes leading to nasal obstruction in children who require prolonged intubation (Greenfeld et al., 2003). However, we should note that in our small cohort, we did not find any association between the length of time that each patient was intubated in the neonatal period and the severity of OSA. All these factors could play a role in the lack of complete resolution of the sleep-associated breathing abnormalities in our children after AT.

This study serves to highlight the symptoms of OSA in former preterm infants. However, several limitations of this report merit discussion. This was a retrospective chart review of a relatively small cohort of children included in a skewed referral population. As such, although current findings are suggestive of the presence of unique risks and adverse outcomes of OSA in this vulnerable population, the true prevalence and clinical features and consequences of OSA in prematurely born infants with and without BPD will have to await a correctly powered and prospectively designed cohort study.

In summary, OSA in infants shares common features of OSA in older children, such as habitual snoring, agitated sleep, and overt apneas. However, we here identify distinct symptoms found in former preterm infants with OSA, and such symptoms include slower than expected weight gain, prolonged need for supplemental oxygen in the context of underlying BPD, or need to re-instate supplemental oxygen after weaning.

sleep disordered breathing? J. Clin. Sleep Med. 6, 264-269.

Capdevila, O. S., Kheirandish-Gozal, L. Dayyat, E., and Gozal, D. (2008). Pediatric obstructive sleep apnea: complications, management, and long-term outcomes. Proc. Am. Thorac. Soc. 5, 274-282.

Chervin, R. D., Archbold, K. H., Dillon, J. E., Panahi, P., Pituch, K. J., Dahl, R. E., and Guilleminault, C. (2002). Inattention, hyperactivity, and symptoms of sleep-disordered breathing. Pediatrics 3, 449-456.

Darrow, D. H., and Siemens, C. (2002). Indications for tonsillectomy and adenoidectomy. Laryngoscope 112, 6-10.

Fajardo, C., Alvarez, J., Wong, A., Kwiatkowski, K., and Rigatto, $\mathrm{H}$. (1993). The incidence of obstructive apneas in preterm infants with and without bronchopulmonary dysplasia. Early Hum. Dev. 32, 197-206. 
Gozal, D. (2008). Obstructive sleep apnea in children: implications for the developing central nervous system. Semin. Pediatr. Neurol. 15, 100-106.

Greenfeld, M., Tauman, R., DeRowe, A., and Sivan, Y. (2003). Obstructive sleep apnea syndrome due to adenotonsillar hypertrophy in infants. Int. J. Pediatr. Otorhinolaryngol. 67, 1055-1060.

Hibbs, A. M., Johnson, N. L., Rosen, C. L., Kirchner, H. L., Martin, R., Storfer-Isser, A., and Redline, S. (2008). Prenatal and neonatal risk factors for sleep disordered breathing in school-aged children born preterm. J. Pediatr. 153, 176-182.

Marcus, C. L., Carroll, J. L., Koerner, C. B., Hamer, A., Lutz, J., and Loughlin, G. M. (1994). Determinants of growth in children with the obstructive sleep apnea syndrome. J. Pediatr. 125, 556-562.

McGowen, F. X., Kenna, M. A., Fleming, J. A., and O'Connor, T. (1992). Adenotonsillectomy for upper airway obstruction carries increased risk in children with a history of prematurity. Pediatr. Pulmonol. 31, 222-226.

Montgomery-Downs, H. E., Crabtree, V. M., Sans Capdevila, O., and Gozal, D. (2007). Infant-feeding methods and childhood sleep-disordered breathing. Pediatrics 120, 1030-1035.

Montgomery-Downs, H. E., and Gozal, D. (2006). Sleep habits and risk factors for sleep-disordered breathing in infants and young toddlers in Louisville, Kentucky. Sleep Med. 7, 211-219.

Montgomery-Downs, H. E., O'Brien, L. M., Gulliver, T. E., and Gozal, D. (2006). Polysomnographic characteristics in normal preschool and early school aged children. Pediatrics 117, 741-753.

Montgomery-Downs, H. E., Young, M. E., Ross, M. A., Polak, M. J., Ritchie, S. K., and Lynch, S. K. (2010). Sleepdisordered breathing symptoms frequency and growth among prematurely born infants. Sleep Med. 11, 263-267.

Paavonen, E. J., Strang-Karlsson, S., Räikkönen, K., Heinonen, K., Pesonen, A. K., Hovi, P., Andersson, S.,
Järvenpää, A. L., Eriksson, J. G., and Kajantie, E. (2007). Very low birth weight increases risk for sleepdisordered breathing in young adulthood: the Helsinki Study of very low birth weight adults. Pediatrics 120, 778-784.

Rechtschaffen, A., and Kales, A. (1968) A Manual of Standardized Terminology, Techniques and Scoring Systems for Sleep Stages of Human Subject, Vol. 204. Washington, DC: National Institutes of Health, 4

Rosen, C. L., Larkin, E. K., Kirchner, H. L., Emancipator, J. L., Bivins, S. F., Surovec, S. A., Martin, R. J., and Redline, S. (2003). Prevalence and risk factors for sleep-disordered breathing in 8- to 11-year-old children: association with race and prematurity. J. Pediatr. 142, 383-389.

Sie, K. C. Y., Perkins, J. A., and Clarke, W. R. (1997). Acute right heart failure due to adenotonsillar hypertrophy. Int. J. Pediatr. Otorhinolaryngol. 41 , 53-58.

Suen, J. S., Arnold, J. E., and Brooks, L. J. (1995). Adenotonsillectomy for treatment of obstructive sleep apnea in children. Arch. Otolaryngol. Head Neck Surg. 121, 525-530.

Conflict of Interest Statement: The authors declare that the research was conducted in the absence of any commercial or financial relationships that could be construed as a potential conflict of interest.

Received: 07 June 2011; accepted: 11 November 2011; published online: 29 November 2011.

Citation: Sharma PB, Baroody F, Gozal $D$ and Lester LA (2011) Obstructive sleep apnea in the formerly preterm infant: an overlooked diagnosis. Front. Neur. 2:73. doi: 10.3389/fneur.2011.00073

This article was submitted to Frontiers in Sleep and Chronobiology, a specialty of Frontiers in Neurology.

Copyright (C) 2011 Sharma, Baroody, Gozal and Lester. This is an open-access article distributed under the terms of the Creative Commons Attribution Non Commercial License, which permits use, distribution, and reproduction in other forums, provided the original authors and source are credited. 